\section{Serge Carillo \\ Magali Pariat \\ Isabelle Jariel-Encontre Ann-Muriel Steff Thierry Lorca Marc Piechaczyk}

\title{
Le catabolisme protéique intracellulaire : une fonction biologique majeure. Partie II: exemples de dégradation conditionnelle et genèse des peptides antigéniques*
}

Le catabolisme protéique joue un rôle majeur dans la cellule, et ne se contente pas d'assurer l'élimination des protéines dont l'accumulation deviendrait toxique. Certaines dégradations sont finement réglées, en particulier celle des facteurs de différenciation et de transcription, des protéines onco-suppressives ( $\mathrm{p53}$ ) et des cyclines mitotiques. Le catabolisme protéique est donc impliqué dans le contrôle d'étapes clés de la différenciation et du cycle cellulaire. En outre, il est à la base de la réponse immune spécifique en permettant l'apprêtement de peptides antigéniques.

es fonctions de la dégradation protéique intracellulaire sont multiples et peuvent être réparties en deux grandes classes: les dégradations non

S. Carillo : docteur en pharmacie, contractuel Bioavenir MESR/Rhône-Poulenc-Rorer M. Pariat, A-M. Steff : boursières $M R$ I. Jariel-Encontre : chargée de recherche au Cnrs. M. Piechaczyk : directeur de recherche au Cnrs. Institut de génétique moléculaire/UMR9942 Cnrs, Route de Mende, BP 5051, 34033 Montpellier Cedex 01, France. T. Lorca : chargé de recherche au Cnrs. CRBM/UPR900, Cnrs, Route de Mende, BP 5051, 34033 Montpellier Cedex 01, France.

TIRÉS À PART

M. Piechaczyk.

* La partie I de cet article est parue dans le précédent numéro de $\mathrm{m} / \mathrm{s}$ ( $\mathrm{n}^{\circ}$ 5, vol. 11 , mai 1995, p. 723-34). $\mathrm{m} / \mathrm{s} n^{\circ} 6$, vol. 11 , juin 95
Nous avons précédemment exposé les principales fonctions et les mécanismes enzymatiques connus du catabolisme protéique [1]. Dans un premier temps, nous présenterons ici une série d'exemples physiologiques de dégradations réglées pour souligner la multiplicité des situations et le fait que l'altération de la dégradation de certaines protéines puisse être associée à des phénomènes pathologiques. Dans un second temps, nous dresserons un tableau général de la production des peptides antigéniques et nous mettrons en évidence que l'ensemble des ressources protéolytiques de la cellule peut être utilisé lors du déclenchement de la réponse immunitaire par un antigène. 


\section{RÉFÉRENCES}

1. Carillo $S$, Pariat, M, Jariel-Encontre I, Steff AM, Piechaczyk M. Le catabolisme protéique intracellulaire: une fonction biologique majeure. Partie I. médecine/sciences gique majeure.

2. Quail PH. Phytochrome: a light-activated molecular switch that regulates plant gene expression. Annu Rev Genet 1991; 25:389. 409.

3. Kellerman KA, Mattson DM, Duncan I. Mutations affecting the stability of the Fushi-tarazu protein in Drosophila. Genes Dev $1990 ; 4: 1936-50$

4. Chowdary DR, Dermody IJ, Jha KK, Ozer HL. Accumulation of p53 in a mutant cell line defective in the ubiquitin pathway. Mol Cell Biol 1994; 14: 1997-2003.

5. Scheffner M, Huibregtse JM, Vierstra RD Howley PM. The HPV-16 E6 and E6-AP complex functions as a ubiquitin-protein ligase in the ubiquitination of p53. Cell 1993 ; 75 : 495-505.

6. Gilmore TD, Morin PJ. IKB proteins : members of a multifunctional family. Trends Genet 1993; 9: 427-33.

7. Henkel T, Machleidt T, Alkalay I, Kronke M, Ben-Neriah Y Bauerle PA. Rapid proteolysis of I $\mathrm{KB}-\mathrm{a}$ is necessary for activation of transcription factor NFKB. Nature 1993; $365: 182-5$.

8. Miyamoto S, Chiao PJ, Verma IM. Enhanced $1 \kappa \mathrm{Ba}$ degradation is responsible for constitutive NF- $\mathrm{KB}$ activity in mature murine B-cell lines. Mol Cell Biol 1994; 14 : 3276 82 .

9. Glotzer M, Murray AW, Kirshner MW. Cyclin is degraded by the ubiquitin pathway. Nature 1991 ; 349 : 132-8.

10. Colombani J. HLA : fonctions immunitaires et applications médicales. Paris: John Libbey Eurotext, 1993.

11. Bahram S. Transporteurs de peptides et présentation de l'antigène. médecine/sciences $1993 ; 9$ : 1204-13.

12. Lotteau V. Restriction dans la présentation des antigènes aux lymphocytes $T$ cytotoxiques. médecine/sciences $1993 ; 9: 1179-81$

13. Lotteau V. Protéasome et présentation de l'antigène. médecine/sciences $1993 ; 9$ 1284-5.

14. Neefjes JJ, Momburg F. Cell biology of antigen presentation. Curr Biol 1993; 5 : 27 34.

15. Rammensee HG, Falk K, Rötzschke $O$. MHC as peptide receptors. Curr Biol 1993;

\section{Exemples de dégradation protéique conditionnelle}

La dégradation du phytochrome est réglée par la lumière chez les plantes

Le phytochrome est une protéine végétale régulatrice impliquée dans la germination, le développement chloroplastique et la floraison. Il existe sous deux formes photoconvertibles. Sa forme de base est stable du point de vue métabolique mais physiologiquement inactive. La transition vers l'état actif (Pfr) est induite par la lumière rouge et s'accompagne de l'accélération du catabolisme. Ce dernier impliquerait une dépense d'énergie et la conjugaison de l'ubiquitine. Il semble donc que, de façon concomitante à l'activation fonctionnelle du phytochrome, la plante mette en place une procédure de contrôle négatif efficace en accélérant la dégradation de cette protéine. Cependant, on comprend mal à l'heure actuelle l'avantage de la protéolyse sur une simple conversion de la forme active vers la forme inactive [2].

Fushi tarazu : un régulateur du développement précoce de la drosophile dont l'action est limitée dans le temps et dans l'espace

La protéine Fushi tarazu (Ftz) est un facteur de transcription essentiel au contrôle du développement précoce de la drosophile. Elle est impliquée dans la formation des segments de l'embryon. En conséquence, les mutations qui interfèrent avec l'activité de Ftz entraînent l'absence de formation des limites segmentaires ainsi que la perte de certaines structures des segments impairs. La protéine $\mathrm{Ftz}$ est très instable avec une demi-vie de 6 minutes. La dégradation consomme de l'énergie et fait probablement intervenir un système protéolytique dépendant de l'ubiquitine. Quelques mutations responsables de la stabilisation et, donc de la suraccumulation de Ftz, ont été étudiées [3]. De façon intéressante, ces mutations en rappellent d'autres préalablement identifiées pour induire un phénotype appelé anti-Ftz caractérisé par le changement d'identité de certains segments et des anomalies des segments pairs. Ces variants Ftz sont dominants sur la protéine sauvage puisqu'en cas de coexpression, ce sont les altérations anti-Ftz qui sont observées. Le mécanisme précis de la stabilisation des mutants n'a pas encore été élucidé. Différentes hypothèses peuvent être avancées et devront être testées pour expliquer l'augmentation de stabilité: (1) une moindre reconnaissance de Ftz par les protéases cellulaires en raison de la mutation des motifs d'instabilité ; (2) l'enfouissement des séquences d'instabilité dans la structure protéique en conséquence d'une mutation à distance altérant la structure globale de la protéine, ou bien encore (3) le renforcement d'une association à des partenaires non identifiés qui, d'une part, "masquerait" Ftz et, d'autre part, amplifierait son effet fonctionnel.

La modification de la dégradation de la protéine oncosuppressive $\mathrm{p} 53$ peut être associée au développement tumoral

Sous sa forme normale, la protéine onco-suppressive p53 semble impliquée dans l'arrêt de la prolifération cellulaire en réponse à l'altération de l'ADN génomique $\left(\mathrm{m} / \mathrm{s} n^{\circ} 2\right.$, vol. 10 , p. 206). Pour cette raison, elle est considérée à l'heure actuelle comme un "gardien du génome" dont l'action donnerait aux systèmes enzymatiques appropriés le temps d'effectuer efficacement la réparation des lésions. La protéine p53 peut aussi être associée au développement tumoral, soit directement, en raison de mutations qui lui confèrent un gain de phénotype, soit indirectement, par réduction de son abondance (figure 1). Dans ce dernier cas, on observe une perte de l'effet anti-prolifératif induit par les lésions de l'ADN. Cette hypothèse trouve un soutien expérimental indirect chez les souris dont les gènes $p 53$ ont été détruits par recombinaison homologue. Les animaux sont viables, en effet, mais développent rapidement des tumeurs variées à forte fréquence (m/s $n^{\circ} 5$, vol. 8, p. 492).

Les mutations du gène $p 53$ représentent l'altération génétique la plus répandue dans les cancers humains, tous types confondus. Certaines de ces mutations sont, non seulement responsables de la perte des proprié- 


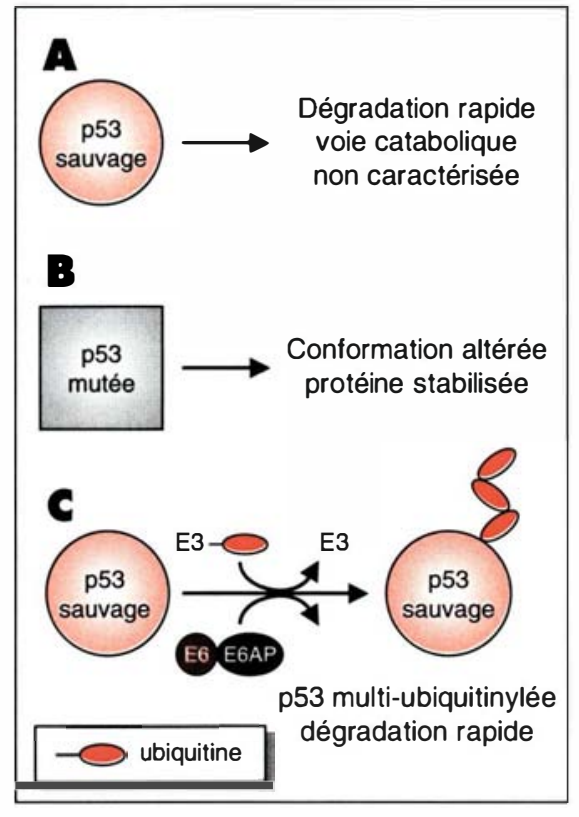

tés anti-tumorales de la protéine, mais aussi de l'apparition de propriétés tumorigènes dont les bases moléculaires exactes restent à définir. La protéine p53 normale est dégradée rapidement (demi-vie de 20 à 40 minutes) par une voie dépendante de l'énergie qui implique probablement l'ubiquitine [3]. De façon frappante, la présence de certaines mutations est associée à une conformation particulière de la protéine ainsi qu'à une forte accumulation intracellulaire. Cette surexpression amplifie l'effet tumorigène et est essentiellement (ou exclusivement) liée au ralentissement de la dégradation de la protéine. L'élucidation des mécanismes impliqués dans la stabilisation, ainsi que la contribution de cette dernière au potentiel tumoral des protéines mutées, fait aujourd'hui l'objet de nombreuses études.

Les papillomavirus sont en général associés à des tumeurs épithéliales squameuses bénines. Cependant, deux d'entre eux, HPV16 et HPV18, sont considérés à haut risque puisque leur présence est liée au développement de carcinomes, en général au niveau de l'utérus. Deux protéines codées par chacun de ces virus, E6 et E7, sont responsables de l'effet oncogénique au travers de l'association à des protéines cellulaires. En particulier, E7 se lie à la protéine onco-sup-

Figure 1. Dégradation de la proteine p53. A. La protéine p53, sous sa forme sauvage, est supposée intervenir dans la répression de la prolifération cellulaire. Elle présente une demi-vie courte inférieure à l'heure. La (les) voie(s) catabolique(s) empruntée(s) est (sont) inconnue(s) bien que le cycle de l'ubiquitine semble impliqué. B. La protéine p53, sous une forme mutée, peut posséder des propriétés oncogéniques. Certaines mutations trouvées dans la partie centrale de la protéine sont responsables d'un changement conformationnel auquel est associé une stabilisation, et donc une suraccumulation, de la protéine dans les cellules tumorales. C. Dans les cellules tumorales infectées par HPV16 et HPV18, la protéine virale E6 s'associe à une protéine cellulaire appellée E6-AP. Le complexe E6/E6-AP reconnaît la protéine p53 à la manière d'une ubiquitine-protéine ligase de type E3. Cette reconnaissance permet l'ubiquitinylation de p53 et accélère la disparition de cette dernière.

pressive $\mathrm{Rb}$ et E6 se lie à p53. Concernant p53, deux caractéristiques de ces tumeurs sont frappantes : la protéine est quasi systématiquement trouvée sous sa forme normale mais son abondance est faible, cela de façon reproductible. Il a pu être montré que l'association E6-p53 accélère la dégradation de p53 par une voie dépendante à la fois de l'ATP et de l'ubiquitine. Cependant, rien ne laisse encore supposer que les mécanismes en cause soient exactement les mêmes que ceux impliqués dans la voie catabolique usuelle de p53. L'étude de la liaison entre p53 et $\mathrm{E} 6$ a révélé l'existence d'un troisième partenaire, E6-AP (E6associated protein) qui ne lie pas p53 en l'absence de E6 mais qui se lie de manière stable à E6 de HPV 16 et 18 en absence de p53. Ces observations, renforcées par le fait qu'E6-AP ne se lie pas aux protéines E6 des HPV à bas risque, confortent donc l'idée que la disparition accélérée de p53 participe au processus tumoral. Des expériences récentes conduites in vitro ont révélé que le complexe E6/E6-AP possède une activité ubiquitine-protéine ligase comparable à celle des enzymes E3 du cycle de l'ubiquitine et favorise la multi-ubiquitinylation de p53 ([4]; pour plus de détails sur les mécanismes, voir [1]).
L'activation du complexe transcriptionnel Rel/NF $\kappa$ B peut être obtenue par dégradation d'un inhibiteur spécifique, IкB

La famille des facteurs de transcription $\mathrm{Rel} / \mathrm{NF} \kappa \mathrm{B}$ est très étudiée en raison de son importance dans la régulation de gènes viraux et de gènes cellulaires impliqués dans la division cellulaire, la différenciation et le développement [5]. Les protéines de cette famille se lient sous forme d'homo- ou d'hétérodimères à des séquences cibles spécifiques (motifs $\kappa \mathrm{B}$ ). L'activité des protéines Rel/NFKB est réglée par une famille d'inhibiteurs spécifiques: $I \kappa B$. Les mécanismes d'action des protéines IKB sont multiples [5]. Ils incluent : (1) l'inhibition de la liaison des complexes Rel/NFKB à l'ADN ; (2) la dissolution de la liaison à l'ADN de ces mêmes complexes et (3) la séquestration du complexe transcriptionnel dans le cytoplasme $\left(\mathrm{m} / \mathrm{s} n^{\circ} 1\right.$, vol. 7, p. 67). Il faut cependant noter que les modes de répression des différents membres de la famille I $\kappa B$ ne sont pas strictement identiques et que la panoplie entière n'est utilisée que par certains d'entre eux. Par ailleurs, dans l'état actuel de nos connaissances, on ne peut pas exclure que le même membre de la famille NFKB soit activé de façon différen- 


\section{RÉFÉRENCES}

16. Sperling AI , Bluestone BA. The firs line of defence. Curr Biol 1993; 3 : 294-6.

17. Elliott T, Smith M, Driscoll P. Peptide selection by class I molecules of the major histocompatibility complex. Curr Biol 1993 3: 854-66.

18. Michalek MT, Grant EP, Gramm C, Goldberg AL, Rock KL. A role for ubiquitin proteolytic pathway in MHC class I restricted antigen presentation. Nature 1993; 363: 552-4.

19. Momburg F, Ortiz-Navarette V, Neefjes II, Goulmy E, Wal Y, Spits H, Powis SJ, Howard JC, Walden P, Hämmerling GJ. The proteasome subunits encoded by the major histocompatibility complex are not essential for antigen presentation. Nature 1992; 360 : $174-7$.

20. Boes B, Hengel H, Ruppert T, Multhaup G, Koszinowski UH, Kloetzel P-M. Interferon $\gamma$ modulates the proteolytic activity and cleavage site preference of $20 \mathrm{~S}$ mouse proteasome. J Exp Med 1994; 179: 901-10.

21. Driscoll J, Brown MG, Finley D, Monaco IJ. MHC-linked LMP genes specifically alter peptidase activity of the proteasome. Nature peptidase activity

22. Gaczynska M, Rock KL, Goldberg AL. $\gamma$ interferon and expression of MHC genes regulate peptide hydrolysis by proteasome. Nature 1993; 365 : 264-7.

23. Heemels MT, Ploegh H. Untapped peptides. Curr Biol 1993; 3: 380-3.

24. Knight SC, Stagg AJ. Antigen-presenting cell types. Curr Op Immunol 1993; 5 : 374-82.

25. Viville S, Rabourdin-Combe C. La chaîne invariante: son rôle et sa fonction dans la réponse immunitaire spécifique. médecine/sciences $1994 ; 10$ : 163-70.

26. Chicz RM, Urban RG, Gorga JC, Vignali AA, Lane WS, Strominger JL. Specificity and promiscuity among naturally processed peptides bound to HLA-DR. I Exp Med $1993 ; 178: 27-47$

27. Harding CV, Collins DS, Slot JW, Geuze HJ, Unanue R. Liposome-encapsulated antigens are processed in lysosomes, recycled and presented to T cells. Cell 1991; 64:393401 .

28. Roche PA, Cresswell P. Proteolysis of the class II-associated invariant chain generates a peptide binding site in intracellular HLADR molecules. Proc Natl Acad Si USA 1991 ; te dans divers contextes physiologiques ou cellulaires.

Dans le cas de l'inactivation de Rel/NFKB par rétention cytoplasmique, différents agents et stimulus extra-cellulaires annulent la latence du facteur de transcription en provoquant le détachement de IкB. Dans le contexte de cette revue, le cas de IкB $\alpha$ est informatif. La plupart des stimulus conduisant à l'activation de $\mathrm{Rel} / \mathrm{NF} \mathrm{B}$ induisent des phosphorylations. Un modèle a donc été régulièrement proposé selon lequel la phosphorylation de $\mathrm{I} \kappa \mathrm{B} \alpha$ induirait, dans un premier temps, sa dissociation de Rel/NFKB, puis, dans un second temps, sa dégradation dans le cytoplasme. Cependant, des observations récentes $[6,7]$ sont en faveur d'un mécanisme différent: (1) à la suite du stimulus, une protéase chymotrypsin-like est activée par phosphorylation et (2) I $\mathrm{KB} \alpha$ est dégradé par cette protéase alors qu'il est encore complexé au dimère Rel/NFkB. Cette protéolyse libère le facteur de transcription qui peut, dès lors, être transporté dans le noyau. Il semble que le protéasome et la voie ubiquitine soient impliqués dans ce processus [8].

La dégradation des cyclines mitotiques est requise pour l'inactivation de la kinase Cdc2 et pour la sortie de la phase $M$ du cycle cellulaire

Les cyclines mitotiques A et B ont été découvertes il y a plusieurs années dans des embryons d'invertébrés marins comme des protéines qui s'accumulent à un niveau élevé avant la mitose et disparaissent rapidement en fin de cette même mitose. Leur synthèse est requise pour la stimulation périodique de la protéine kinase Cdc2 à laquelle elles s'associent pour permettre la condensation des chromosomes, la mise en place du fuseau mitotique et la rupture de l'enveloppe nucléaire. Différentes cyclines, intervenant dans d'autres phases du cycle cellulaire ont, par la suite, été caractérisées. Des systèmes acellulaires performants, permettant de reproduire fidèlement ces événements in vitro, ont été mis au point à partir d'œufs de xénope. Ils ont permis de montrer que la seule synthèse protéique nécessaire au maintien des oscillations entre les états interpha- sique et mitotique est celle de la cycline B. Comme il a déjà été mentionné dans la première partie de cette revue [1], il existe dans la région $\mathrm{N}$ terminale de l'ensemble des cyclines connues un motif de 9 acides aminés (RXALGX'IXN)* relativement conservé appelé «boîte de destruction" du fait de son implication dans le catabolisme de ces protéines. Il comprend 2 acides aminés invariants ( $\mathrm{R}$ et $\mathrm{L}$ ), 4 autres relativement conservés (A, $\mathrm{G}$, I et $\mathrm{N}$ ) et un résidu (X') qui permet de différencier les cyclines A et B. X' est une valine pour les cyclines $A$ et un acide glutamique ou aspartique pour les cyclines $B$. Sa délétion ou la mutation de l'arginine $(R)$ en alanine confèrent l'indestructibilité à la cycline B. Les travaux de Glotzer et al. [9] ont montré que la cycline B est polyubiquitinylée durant la mitose et que cette modification est responsable de la protéolyse spécifique lors de l'anaphase. Lorsque l'ARN messager codant pour une cycline mutée sur l'arginine de la boîte de destruction est ajouté à des extraits d'ovocytes de xénope interphasiques, sa traduction induit, comme prévu, l'entrée en mitose. Toutefois, la cycline B ne peut pas être dégradée et la protéine kinase Cdc2 est maintenue dans son état activé. En outre, l'extrait reste bloqué en mitose au lieu de retourner en interphase, ce qui démontre que la dégradation des cyclines est bien l'événement qui déclenche l'inactivation de Cdc2 et la sortie de mitose.

Par ailleurs, il est intéressant de noter que l'activation périodique de la dégradation de la cycline $B$ est contrôlée par l'activité kinase Cdc2 elle-même (figure 2). Lorsque le complexe fonctionnel Cdc2-cycline B est ajouté à un extrait interphasique d'ovocytes de xénope, c'est-à-dire un stade où la machinerie de protéolyse spécifique est silencieuse, l'extrait est conduit à un stade correspondant à l'anaphase, moment où la machinerie protéolytique est à son maximum d'efficacité.

Lors de la phase $\mathrm{M}$, les cyclines sont phosphorylées par les protéines

\footnotetext{
* Arg-X-Ala-Leu-Gly-X'Ileu-X-Asn où X représente n'importe quel acide aminé.
} 


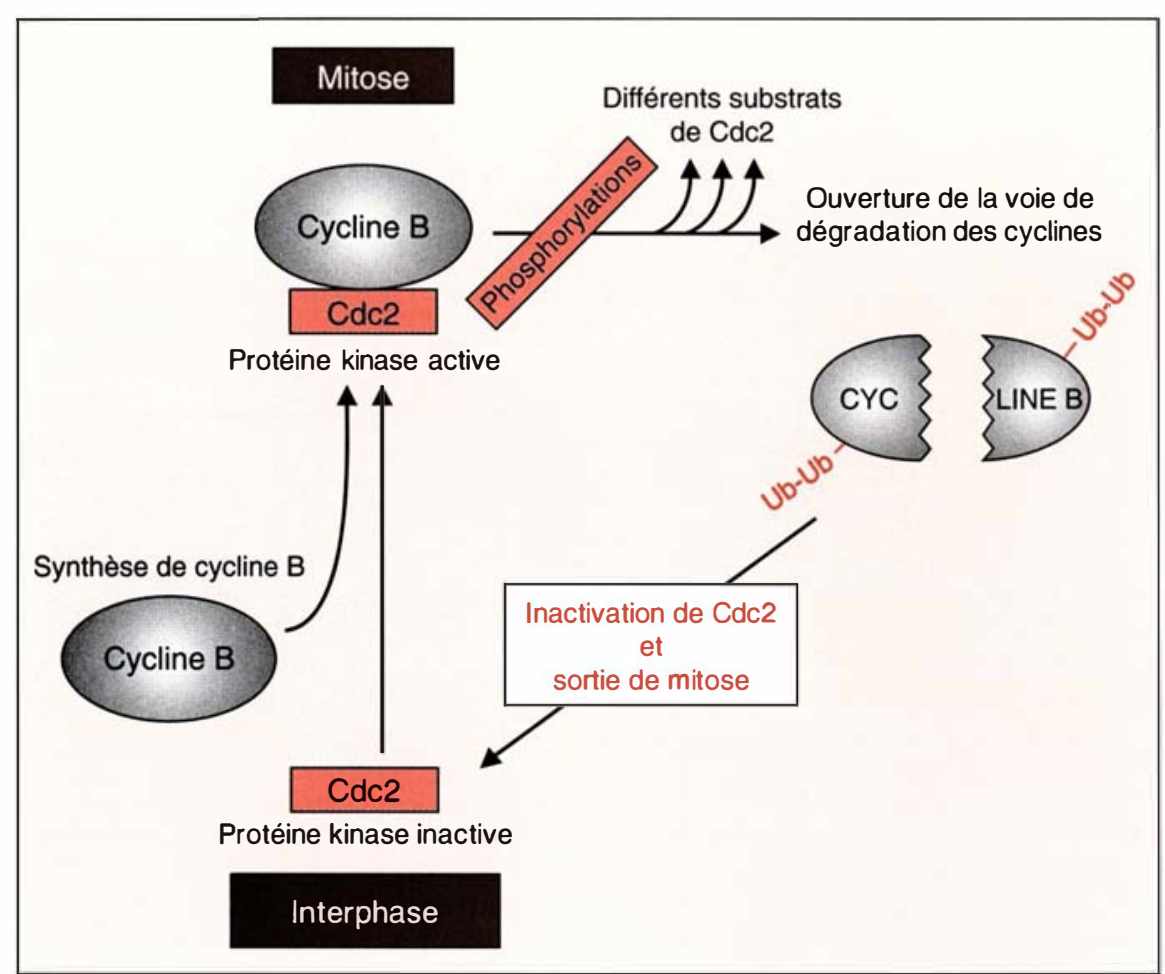

Figure 2. L'inactivation de la protéine kinase mitotique Cdc2 requiert la dégradation de sa sous-unité régulatrice, la cycline $B$. La cycline $B$ est synthétisée en phase G2 du cycle cellulaire. Son association à la protéine Cdc2 stimule l'activité kinase de cette dernière et permet l'entrée en phase $M$. Le complexe Cdc2-cycline active aussi la machinerie de dégradation des cyclines et par voie de conséquence sa propre inactivation. La synthèse, lors de la phase G2, et la dégradation, lors de la phase $M$, des cyclines sont deux événements absolument nécessaires pour permettre les oscillations entre états interphasique et mitotique.

kinases Cdc2 et MAPK (mitogen activated protein kinase). Toutefois, ces phosphorylations ne semblent pas intervenir dans le processus de dégradation. En effet, la mutation des principaux sites de phosphorylation des cyclines n'affecte pas leur destruction.

Comme il a été mentionné plus haut, il existe d'autres cyclines $(\mathrm{C}, \mathrm{D}, \mathrm{E}, \mathrm{F}$, $\mathrm{G}$ et $\mathrm{H}$ ) appelées ainsi en raison de leur analogie de séquence avec les cyclines A et B et/ou de leur association avec des sous-unités catalytiques Cdk (cyclin-dependent kinase). Elles interviennent principalement avant et après la phase M. Jusqu'à présent, l'implication de la voie ubiquitine dans leur dégradation n'a pas été établie ou étudiée. Enfin, chez la levure Schizosaccharomyces pombe, certains homologues de ces cyclines sont rapidement dégradés en fin de phase $G_{1}$. La délétion des séquences PEST pré- produire des peptides antigéniques qui sont présentés par les molécules du CMH. Le second est que la plupart des mécanismes mis en jeu ne sont pas spécialisés dans la genèse de peptides antigéniques: la production de peptides présentables par les molécules de CMH est peut-être pour l'essentiel une simple conséquence du "ménage cellulaire".

Les molécules de classe I, trouvées sur la plupart des types cellulaires, présentent des peptides d'origine endogène (mais pas toujours, voir plus bas), c'est-à-dire engendrés dans la cellule à partir de protéines cellulaires ou de parasites intracellulaires comme les virus. Leur fonction est de permettre la reconnaissance par des lymphocytes $\mathrm{T}$ cytotoxiques, soit de cellules infectées, soit de cellules présentant des épitopes du soi modifiés (ayant subi une activation oncogénique, par mutation par exemple). Les molécules de classe II ne sont, en revanche, présentes que sur certaines cellules du système immunitaire [10]. Elles s'associent à des peptides dérivant de protéines endogènes ou d'origine extracellulaire. Elles sont impliquées dans le déclenchement et le contrôle de la réponse immune. A côté des molécules de classe I dites "classiques", existent aussi des molécules dites "non classiques". Elles sont très peu polymorphes et essentiellement trouvées sur certaines cellules épithéliales, en particulier intestinales. Elles constituent probablement une première ligne de défense contre les infections microbiennes. Il semble qu'elles fixent, à la surface des cellules, des peptides de l'environnement produits par des protéases extracellulaires [16]. Leur cas ne sera donc pas détaillé ici.

L'un des points de contrôle de la réponse immune réside dans la capacité des lymphocytes $\mathrm{T}$ de répondre aux antigènes présentés à la surface d'autres cellules par les molécules polymorphes du complexe majeur d'histocompatibilité de classe I (CMH-I), ou de classe II (CMH-II). Il est évidemment hors de propos d'effectuer ici une revue exhaustive de la présentation antigénique. Pour cela, le lecteur pourra se référer à de nombreuses revues [10-15] parues, en particulier dans médecine/sciences. Notre propos se limitera à souligner deux points. Le premier est la multiplicité des mécanismes utilisés pour
Les molécules CMH-I sont composées de deux polypeptides membranaires: naire présentratrice de l'antigène, codée dans le locus CMH-I, et une molécule extracellulaire associée, la $\beta 2$-microglobuline. Les deux protéines sont synthétisées au niveau du réticulum endoplasmique où elles s'associent pour former un hétérodimère instable à température physiologique. La structure est ensuite stabilisée par la liaison d'un peptide présentant la structure et la taille adéquates. Presque tous les peptides associés au CMH-I qui ont été une chaîne lourde $(\mathrm{H})$ transmembra- 
Figure 3. Genèse et présentation des peptides par les molécules du $\mathrm{CMH}$ I. A. Transport dépendant des protéines TAP. Les protéines cytosoliques et nucléaires sont dégradées en peptides par les systèmes protéolytiques du cytosol. Le protéasome $20 S$ et les protéinases 265 sont probablement les enzymes qui interviennent de façon prépondérante. Les produits de dégradation sont transloqués dans le lumen du réticulum endoplasmique grâce à un transporteur constitué de deux protéines, TAP1 et TAP2. Leur dégradation est probablement poursuivie jusqu'à une taille leur permettant d'être accommodés par le sillon des molécules du CMH-I. De façon concomitante, la chaîne lourde de classe l et la $\beta 2$-microglobuline sont synthétisées et s'assemblent. Après fixation, les extrémités du peptide sont peut-être "grignotées" un peu plus pour lui permettre une présentation optimale. Les hétérotrimères sont ensuite transportés à travers le Golgi vers la membrane cellulaire. B. Présentation d'antigènes indépendamment du système TAP. Les molécules du $\mathrm{CMH}-\mathrm{I}$ vides sont internalisées par endocytose. Parallèlement, des protéines cytoplasmiques sont séquestrées par autophagie dans une structure qui, après fusion avec le compartiment endocytosique, libère des peptides susceptibles de se lier aux molécules du $\mathrm{CMH}-\mathrm{I}$. Après fusion de l'endosome avec la membrane cellulaire, les molécules de $\mathrm{CMH}-\mathrm{l}$ chargées en peptides sont à nouveau exposées.

séquencés sont des peptides du soi, correspondant à des protéines cytosoliques ou nucléaires [17]. Une notion importante à retenir est donc que la grande majorité d'entre eux ne sont pas antigéniques à proprement parler mais simplement «présentables». Le modèle qui domine actuellement pour expliquer la genèse et la présentation des peptides par les CMH-I stipule que : (1) des peptides sont produits à partir de protéines dégradées dans le cytoplasme ; (2) ces peptides, dont la taille et la nature sont inconnues, sont transportés dans le réticulum endoplasmique grâce à un transporteur spécifique dont les deux sous-unités, TAP-1 et TAP-2, sont codées par des gènes

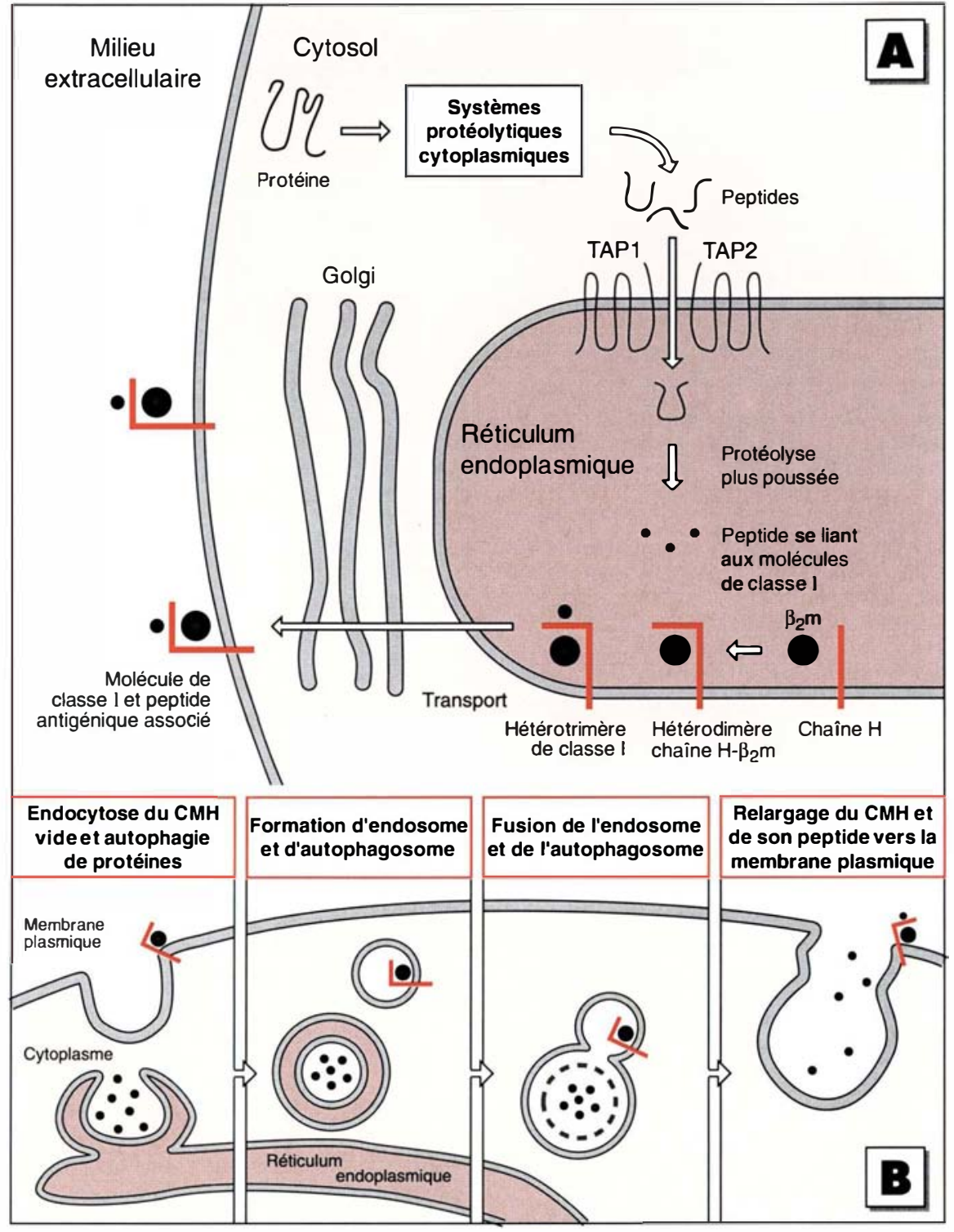

situés dans le locus CMH [11] ; (3) la dégradation des peptides est éventuellement poursuivie dans ce compartiment ; (4) les peptides présentant la structure adaptée s'associent au dimère $\mathrm{H} / \beta 2$-microglobuline et (5) la maturation des peptides est ensuite achevée par des exopeptidases pour les ramener à une taille de 8 à 11 acides aminés, optimale pour une bonne présentation. Les hétérotrimères ainsi formés sont ensuite acheminés au travers de l'appareil de Golgi vers la membrane cellulaire où les peptides sont éventuellement reconnus par des lymphocytes $\mathrm{T}$ cytotoxiques (figure $3 A$ ). Le protéasome a déjà été impliqué dans la genèse des peptides antigéniques
[10], de même que la voie ubiquitine [18]. De ce point de vue, il est intéressant de constater que deux protéines constitutives du protéasome, Lmp2 et Lmp7, sont codées par des gènes situés eux aussi dans le locus CMH-II (les gènes $l m p 2$ et $l m p 7$ sont, en fait, imbriqués avec les gènes TAP 1 et $T A P-2)$. Bien que cette co-localisation ait soulevé l'hypothèse d'une relation fonctionnelle, il a été démontré que $\operatorname{lmp} 2$ et $\operatorname{lmp} 7$ ne sont pas absolument nécessaires à la genèse de peptides antigéniques [19]. Les gènes $l m p$-2 et $l m p$-7 (de même que les gènes TAP) étant inductibles par l'interféron $\gamma$, c'est-à-dire par la réponse inflammatoire, la possibilité d'un rôle plus subtil de ces deux pro- 
téines a été avancée: elles modifieraient la nature du répertoire et la quantité de peptides présentables. Cette hypothèse a récemment trouvé un support expérimental. La présence de Lmp2 et Lmp7 favoriserait, en effet, les coupures endoprotéolytiques par le protéasome après les acides aminés hydrophobes et basiques [20-22]. La plupart des peptides présentés par CMH-I présentant à leur extrémité C-terminale ce type de résidu, il est tentant de supposer que Lmp2 et Lmp7 augmenteraient la production des peptides chargeables par les CMH-I. Il est aussi important de souligner que rien, à l'heure actuelle, n'exclut que d'autres protéinases cytoplasmiques puissent intervenir dans le processus. A côté de ce mécanisme de genèse et de présentation (probablement majoritaire et souvent le seul à être pris en compte dans la littérature), il en existe au moins deux autres [23]. Le premier correspond à la régurgitation de peptides produits par les cellules phagocytaires suivie de leur récupération par endocytose par des cellules non phagocytaires du voisinage. Il s'agit d'une véritable coopération cellulaire. C'est probablement de cette manière que les cellules dendritiques (cellules non phagocytaires impliquées dans les phases précoces de la réponse immune) [24] mettent en route la réponse immunitaire contre les agents microbiens phagocytés et dégradés par les macrophages (figure 3B). Le second mécanisme correspond à l'association des molécules de CMH-I avec des peptides engendrés dans le compartiment lysosomial. Ce scénario tire parti du fait que beaucoup de peptides sont associés de façon relativement lâche aux molécules de CMH-I et sont, pour cette raison, facilement relargués dans le milieu extracellulaire. Après endocytose, les vésicules endosomiques contenant des molécules de CMH-I libres peuvent fusionner avec des autophagosomes. Ainsi, ces dernières peuvent-elles être rechargées en peptide et à nouveau routées vers la membrane plasmique. Les molécules de CMH-II présentent des similitudes avec celles de CMH-I dans le sens où elles sont dimériques et que leurs deux chaînes peptidiques constitutives, $\alpha$ et $\beta$, sont toutes deux codées par des gènes situés dans le même locus génomique. Il existe cependant des différences parmi lesquelles on peut noter l'association avec des peptides beaucoup plus longs (12-25 acides aminés) que ceux présentés par les CMH-I ainsi que l'association intracellulaire à une troisième chaîne transmembranaire ou chaîne invariante (Ii) [25]. Il est encore souvent avancé que les seuls peptides présentés par CMH-II sont engendrés à par- tir de protéines exogènes internalisées par endocytose. Il s'agit d'un concept erroné. En fait, tout comme pour les CMH-I, presque tous les peptides associés aux CMH-II qui ont été séquencés jusqu'à aujourd'hui $[15,26]$ se sont révélés être d'origine intracellulaire (dérivant souvent de $\mathrm{CMH}$ ou de molécules associées). Le modèle actuel pour la genèse et la présentation des peptides d'origine exogène par les $\mathrm{CMH}-\mathrm{II}$ est le suivant (figure 3): (1) les chaînes $\alpha$, $\beta$ et Ii sont synthétisées au niveau du réticulum endoplasmique où elles s'associent suivant un mécanisme qui reste à découvrir ; (2) le rôle de Ii est probablement double. Elle empêche, d'une part, l'association des CMH-II avec des peptides du réticulum endoplasmique et elle augmente, d'autre part, l'efficacité du transport du complexe $\alpha / \beta$ vers le réticulum transGolgien au travers de l'appareil de Golgi ; (3) à ce niveau, les complexes sont déviés vers la voie endocytosique grâce à un motif présent dans la partie cytoplasmique de Ii ; (4) le site précis où les CMH-II rejoignent la voie endocytosique n'est pas encore clairement établi puisque ces molécules ont été retrouvées à la fois dans les endosomes précoces et tardifs et dans les lysosomes ; (5) il semble néanmoins que le site majeur de genèse des peptides antigéniques et de leur association avec les CMH-II

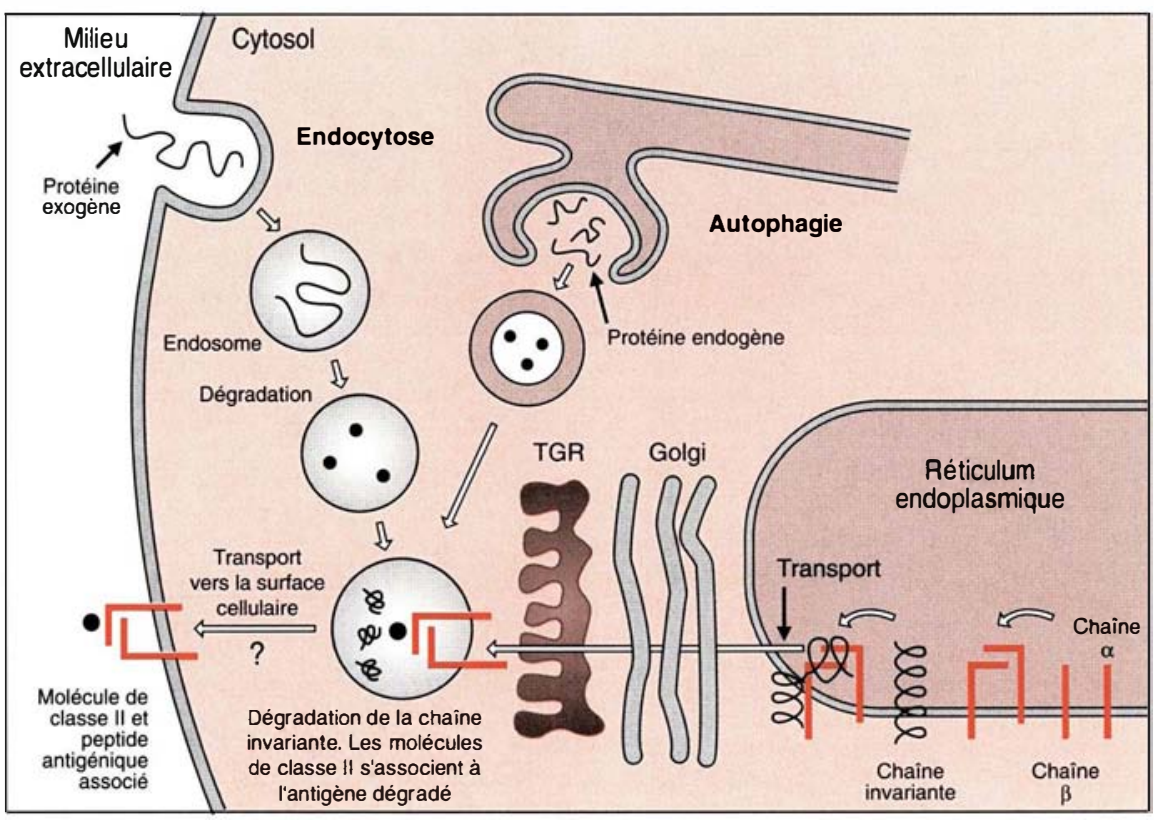

$\mathrm{m} / \mathrm{s} n^{\circ} 6$, vol. 11 , juin 95
Figure 4. Genèse et présentation des peptides par les molécules du CMHII. Les chaînes $\alpha$ et $\beta$ du CMH-Il ainsi que la chaîne invariante li sont synthétisées dans le réticulum endoplasmique. Un nonamère composé de 3 $\alpha, 3 \beta$ et 3 li est alors formé. In empêche la fixation de peptides dans ce compartiment. Les complexes sont alors véhiculés vers le compartiment endocytosique tardif grâce à li via le réseau trans-golgien (TGR). li y est dégradée, permettant ainsi la captation de peptides par les dimères $\alpha / \beta$ et le routage du nouveau trimère formé vers la membrane cellulaire. Alternativement, les complexes $\alpha / \beta / l i$ peuvent être dirigés vers les autophagosomes et permettre la présentation de peptides d'origine intracellulaire. 
soit le lysosome [27] ; les cathepsines $\mathrm{D}$ et $\mathrm{E}$ joueraient un rôle crucial dans ce processus ; (6) la liaison du peptide s'effectue à la faveur de la séparation et de la dégradation d'Ii mise en route par la cathepsine B [28] et (7) les CMH-II chargés sont dirigés vers la membrane cytoplasmique. Bien que peu d'attention ait été portée à la genèse de peptides à partir de protéines endogènes et à leur présentation par les CMH-II, il est vraisemblable que l'autophagie joue un rôle crucial dans le processus. Les modalités de l'interaction entre $\alpha, \beta$ et Ii étant, d'une part, inconnues et la présence de Ii n'étant, d'autre part, pas absolument indispensable au routage du dimère $\alpha / \beta$, il n'est pas non plus exclu qu'une fraction des $\mathrm{CMH}$ II puisse fixer des peptides endogènes dès le réticulum endoplasmique.

En conclusion, l'ensemble des ressources protéolytiques de la cellule peut être mis à profit pour produire des peptides présentables par les molécules du complexe majeur d'histocompatibilité. Dans le cas des
CMH-II, le lysosome permet la production de ces fragments peptidiques, aussi bien à partir de protéines d'origine externe que de protéines d'origine interne. Dans le cas des CMH-I, les systèmes protéolytiques cytoplasmiques, comme le protéasome, sont largement responsables de la genèse de ces peptides à partir de protéines intracellulaires. Il ne s'agit cependant pas d'un mécanisme exclusif puisque les lysosomes peuvent aussi être impliqués. Enfin, il faut prendre en compte que des peptides présentables régurgités par des cellules phagocytaires ont la possibilité d'être récupérés par endocytose à partir du milieu extracellulaire

\section{Remerciements}

Nous remercions les Dr F. Martin et U. Hibner pour leur lecture critique du manuscrit et $S$. Batigne pour l'aide apportée aux figures. Les travaux effectués dans notre laboratoire ont été financés par le Cnrs, l'ARC, la Ligue contre le cancer, l'ANRS et le projet Bioavenir MRT/Rhône-Poulenc-Rohrer.

\section{Summary}

Intracellular protein catabolism : a major biological function. Part II : examples of conditional degradation and genesis of antigenic peptides

Protein catabolism is of major importance for cell physiology. It is responsible for the elimination of peptides, the accumulation of which would turn out to be toxic. It is also involved in the control of key steps of cell differentiation and proliferation. Moreover, it is at the origin of the specific immune response through the production of antigenic peptides. We have previously detailed the intimate mechanisms of protein degradation $\left(m / s n^{\circ} 6\right.$, vol. 11, p. 723). Herein, we present examples of regulated degradation along with the processes involved in the production of peptides presentable by the major histocompatibility complex molecules. 